\title{
Amplification of localized acoustic waves by the electron drift in a quantum well
}

\author{
A. A. Demidenko, V. A. Kochelap \\ Institute of Semiconductor Physics, National Academy of Sciences of Ukraine, Kyiv, 252028, Ukraine
}

\begin{abstract}
We have investigated acoustic waves in a heterostructure with a layer embedded into a semiconductor providing acoustic waves localization near the layer and electron confinement inside the layer. For layer thicknesses smaller than wavelengths we have obtained and analyzed the dispersion relation for the localized waves. For electrons into the layer we have supposed that parallel transport is semiclassical, while perpendicular electron motion is quantized. For two-dimensional confined electrons interacting with the acoustic waves we have solved the Boltzmann equation in a parallel electric field. The solutions have been found for electron-phonon interaction via deformation potential. The dispersion relation for coupled charge density and acoustic waves has been analyzed. We have established conditions of amplification of localized acoustic waves under the electron drift for two extreme cases: i) the only lowest two-dimensional subband is populated, ii) a large number of the subbands are populated. We have found that the amplification coefficient of the acoustic waves in THz-rigion is of the order of $100 \mathrm{~cm}^{-1}$. We have discussed the results and compared them with acoustic waves amplification in bulk like semiconductors.
\end{abstract}

Keywords: acoustic waves localization, acoustic waves amplification, electron drift.

Paper received 21.01.99; revised manuscript received 17.05.99; accepted for publication 24.05.99.

\section{Introduction}

The phenomena of sound amplification and generation by the charge carrier drift [1] have been studied in detail since the beginning of the 60's [2], mainly in uniform bulk semiconducting materials (see, for example, the reviews [3-6]). Different mechanisms of electron-phonon interactions were considered. These include: piezoelectric interaction, interactions via the deformation potential, the electrostriction mechanism [7], etc. During the same period, the general laws of sound amplification and generation were established. Multilayer and spatially bounded materials were also studied. In these cases, the specific features of the formation of sound waves were taken into account, particularly, the possibility of their localization in separate layers or near the surfaces $[8,9]$. The thickness of the layers was assumed to be larger than a micrometer size, and electrons in all cases without any exceptions were considered as three-dimensional.

Quantum heterostructures, which are now the most popular object of studies in the semiconductor physics, consist of layers having dimensions of the order of a hundred of Angstroms or less, and their electron subsystems are essentially quantized [10]. These two conditions are essential both for the electron-phonon interactions in general (see, for example, [11]), and for the acous- 


\section{A. A. Demidenko et al.: Amplification of localized acoustic waves by ...}

tic-electronic phenomena in particular. The latter include interactions of electrons with sound flows, dragging of electrons by phonons, amplification and generation of sound by electrons.

Interactions of electrons with acoustic vibrations in quantum heterostructures were studied, first of all, as one of mechanisms of electron scattering, which controls the low-field mobility and relaxation of low-dimensional hot electrons [11-13]. Two types of lattice vibrations, quasi-bulk and those localized in the region of the electron confinement, were taken into account. The role of such interactions was found to increase with reduction of the electron dimensionality. In the heterostructures based on III-V semiconductors the acoustic phonons may control the electron relaxation of two- and one-dimensional electrons up to the liquid nitrogen temperatures in the electric field range of 100 to $200 \mathrm{~V} / \mathrm{cm}$

The interaction of electrons with sound flows was used in [14-17] both to determine the peculiarities of the electron-phonon interaction and to test such specific lowdimensional effects as the quantum Hall effect, etc. Generally, there are few works devoted to acoustic-electronic phenomena. To a great extent, this is associated with the following. For typical experimental conditions, bulk (or surface) waves are excited. The electrons are localized within the quantum wells and wires. The spatial zones of overlapping of the sound waves and electrons are very narrow and, as a consequence, the effect of electrons on the propagation of such waves is weak.

The latter fact is even more critical for the amplification and generation of acoustic waves. To demonstrate it, let us consider a semiconductor structure having the dimensions $L_{x} \times L_{y} \times L_{z}$ with the electrons localized in a quantum well perpendicular to the $Z$ axis. When calculating the amplification (absorption), it is necessary to consider the interaction of electrons with each acoustic mode. For a mode propagating along the $X$ axis, evidently, amplification (absorption) will be proportional to $\left(d / L_{z}\right)$ where $d$ is the width of the quantum well. As $d<<L_{z}$, amplification (absorption) of the bulk mode is extremely low. Thus, we come to the conclusion that the bulk modes can not be significantly amplified (or attenuated) by the electrons.

To achieve amplification of an acoustic wave, it should be localized in the layer where electrons are quantized. This type of localization is possible in two cases: (1) due to a difference between elastic properties of the quantum well layer and the adjacent layers [18-20], and (2) due to the electron-phonon interaction, which localizes the acoustic wave even in the conditions of initially uniform elastic properties of the structure [21-23]. In both cases, the degree of localization of vibrations near the quantum well layer increases with the vibration frequency, which should result in increasing the amplification factor for high-frequency acoustic waves. It will be shown below that efficient amplification of acoustic waves by the electron drift in the quantum wells is achieved in the frequency range above $100 \mathrm{GHz}$.
It should be noted that an experimental technique for detecting acoustic vibrations with frequencies of the order of hundreds $\mathrm{GHz}$ has been already developed [24, 25]. At present, such oscillations are generated using pulse techniques $[24,25]$ : an irradiation with an ultrashort (subpicosecond) laser pulse results in thermal stresses in the area of light absorption, which then induces a coherent (non-monochromatic) high-frequency acoustic signal propagating in the material.

In this paper, it is suggested to amplify the high-frequency acoustic wave localized near the quantum well by the electron drift. A calculation is presented of the amplification (absorption) coefficient and of the wave localization parameter for the electron-phonon interaction via the deformation potential.

In section II, free localized acoustic waves and those induced by the variable electron density $n(\vec{r}, t)$ are considered. Section III discusses the interaction of electrons in quantum and classical wells (layers) with the acoustic waves. The function $n(\vec{r}, t)$ is specified, and the self-consistent solutions for the electron-phonon system under study are obtained. Numerical estimations of the coefficient of amplification (absorption), parameter of localization, and renormalization of the sound velocity are presented. The main results are summarized in Section IV, while Appendixes I-III present some auxiliary results.

\section{Localized acoustic waves}

Assume that a continuous isotropic medium contains a layer with a thickness of $2 d$, perpendicular to the $\mathrm{Z}$ axis, whose properties are different from those in the bulk. Let the first Lame constans are $\lambda$ inside the layer and $\bar{\lambda}$ outside. Further on, all parameters outside the layer will be denoted by the same letters as those inside, but with a dash above. For the sake of simplicity, to prevent a mixing of the longitudinal and transverse solutions, we assume for the second Lame constant $\bar{\mu}=\mu$. We also assume that the dielectric constant $\bar{\varepsilon}=\varepsilon$, and the density

of the material is $\bar{\rho}=\rho$. Let us denote $\frac{\bar{\lambda}+2 \mu}{\lambda+2 \mu}=h$.

The concentration of charge carriers in the layer consists of the equilibrium term $N(z)$ and a nonequilibrium deviation $n(x, z, t)=n(z) e^{i q x-i \omega t}$ The same dependence on $x$ and $t$ is assumed for all variables. In this section, we assume $n(z)$, and, hence, the right-hand sides of the equations, to be specific functions of $z$. The dependence $n(z)$ will be specified in the next section. Here, we assume only that $n(z)$ is an even function of $z$ vanishing at the layer boundaries: $n( \pm d)=0$. Inside the layer, the variable part of the stress tensor contains an additional term $\sigma_{i k}^{\prime}=\delta_{i k} b n$, where $b$ is the constant of the deformation potential.

Consider a longitudinal elastic wave whose vector of the elastic displacement $\vec{U}$ has two components $U_{x}, U_{z}$ depending on $x, z, t$. The problem is assumed to be uniform with respect to $y$. 


\section{A. A. Demidenko et al.: Amplification of localized acoustic waves by ...}

The equations of motion of the medium inside the layer $(|z|<d)$, have the following form (the accent denotes the derivative with respect to $z$ ) [27]:

$$
\begin{aligned}
& k_{L}^{2} q_{T}^{2} U_{x}-q_{L}^{2} U_{x}^{\prime \prime}-i q\left(q_{T}^{2}-q_{L}^{2}\right) U_{z}^{\prime}=i q \beta n, \\
& k_{T}^{2} q_{L}^{2} U_{z}-q_{T}^{2} U_{z}^{\prime \prime}-i q\left(q_{T}^{2}-q_{L}^{2}\right) U_{z}^{\prime}=\beta n^{\prime}
\end{aligned}
$$

where:

$$
\begin{gathered}
q_{L}^{2}=\frac{\omega^{2}}{V_{L}^{2}}, V_{L}=\sqrt{\frac{\lambda+2 \mu}{\rho}}, q_{T}^{2}=\frac{\omega^{2}}{V_{T}^{2}} \\
V_{T}=\sqrt{\frac{\mu}{\rho}}, k_{L, T}^{2}=q^{2}-q_{L, T}^{2}, \beta=\frac{b q_{T}^{2}}{\lambda+2 \mu}
\end{gathered}
$$

Outside the layer $(|z|>d)$ the equations are the same but the right-hand sides are equal to zero $(\bar{n}=0)$, and the substitution $q_{L}^{2} \rightarrow \bar{q}_{L}^{2}=\frac{1}{h} q_{L}^{2}$ should be made.

Elimination of $U_{x}$ from (1), (2) yields the equation for $U$ :

$$
U_{z}^{\prime \prime \prime \prime}-\left(k_{L}^{2}+k_{T}^{2}\right) U_{z}^{\prime \prime}+k_{L}^{2} k_{T}^{2} U_{z}=-\frac{\beta}{q_{T}^{2}}\left(n^{\prime \prime \prime}-k_{T}^{2} n^{\prime}\right)
$$

From a general solution of Eq.(3), we choose the solution matching in parity the right-hand side (solutions of the opposite parity will not interact with the carriers):

$$
U_{z}(z)=A \sinh k_{L} z+B \sinh k_{T} z+y(z),
$$

where

$$
y(z)=\sum_{i=1}^{4} \frac{e^{k_{i} z}}{P^{\prime}\left(k_{i}\right)} \int_{0}^{z} e^{-k_{i} z}\left[-\frac{\beta}{q_{T}^{2}}\left(n^{\prime \prime \prime}-k_{T}^{2} n^{\prime}\right)\right] d z,
$$

$k_{i}= \pm k_{L}, \pm k_{T}-$ are the roots of the characteristic polynomial of Eq.(3)

$$
P(k)=k^{4}-\left(k_{L}^{2}+k_{T}^{2}\right) k^{2}+k_{L}^{2} k_{T}^{2}
$$

The calculation of $\mathrm{y}(\mathrm{z})$ is presented in Appendix 1:

$$
\begin{aligned}
& y(z)=\frac{\beta}{q_{T}^{2}}\left[\frac{n^{\prime \prime}(0)}{q_{T}^{2}-q_{L}^{2}}\left(\frac{\sinh k_{L} z}{k_{L}}-\frac{\sinh k_{T} z}{k_{T}}\right)+\right. \\
& \left.+n(0) \frac{\sinh k_{L} z}{k_{L}}-\frac{1}{2} J_{+}\left(k_{L}, z\right)\right] .
\end{aligned}
$$

The definitions and properties of the integrals $J_{ \pm}$are described in Appendix 1. Substitution of (4) in (2) followed by integration gives the expression for $U_{x}$ :
$U_{x}(z)=\frac{i}{q}\left[\frac{q^{2}}{k_{L}} A \cosh k_{L} z+k_{T} B \cosh k_{T} z+\Psi(z)+C\right]$,

where $\Psi(z)=\frac{1}{q_{T}^{2}-q_{L}^{2}}\left[-k_{T}^{2} q_{L}^{2} \int_{0}^{z} y(z) d z+q_{T}^{2} y^{\prime}+\beta n\right]$.

The constant $C$ is determined by substitution of (4), (7) into (1). According to (6)

$\Psi(z)+C=\frac{\beta}{q_{T}^{2}}\left[\frac{n^{\prime \prime}(0)}{q_{T}^{2}-q_{L}^{2}}\left(\frac{q^{2}}{k_{L}^{2}} \cosh k_{L} z-\cosh k_{T} z\right)+\right.$

$\left.+\frac{q^{2}}{k_{L}^{2}} n(0) \cosh k_{L} z-\frac{1}{2} \frac{q^{2}}{k_{L}} J_{-}\left(k_{L}, z\right)\right]$.

Outside the layer $(|z|>d)$ we choose the solutions decaying at $z \rightarrow \pm \infty$ :

$$
\begin{aligned}
& \bar{U}_{z}=\frac{z}{|z|}\left(\bar{A} e^{-\bar{k}_{L}|z|}+\bar{B} e^{-k_{T}|z|}\right), \\
& \bar{U}_{x}=-\frac{i}{q}\left(\frac{q^{2}}{\bar{k}_{L}} \bar{A} e^{-\bar{k}_{L}|z|}+k_{T} \bar{B} e^{-k_{T}|z|}\right),
\end{aligned}
$$

The four constants $A, \bar{A}, B, \bar{B}$ are determined by the equations of boundary conditions (BC) at the layer boundaries. The set of $\mathrm{BC}$ equations and its solution are given in Appendix 2.

If the determinant of the system is not equal to zero, then the equations determine the variables $U_{x}, U_{z}, \bar{U}_{x}, \bar{U}_{z}$ in the form of quadratures of $n(z)$. In our model, carriers interact only with the longitudinal waves, so we present here the expressions for $\operatorname{div} \bar{U} \equiv W(z)$ only, calculated in Appendix 2:

$$
\begin{aligned}
& W(z)=-\frac{q_{L}^{2}}{k_{L}} A \cosh \left(k_{L} z\right)+y^{\prime}-\Psi-C= \\
& =-\frac{b q_{L}^{2}}{2(\lambda+2 \mu)} \frac{J_{+}\left(k_{L}, d\right) \cosh \left(k_{L} z\right)}{F(\omega, q)} ; \\
& \bar{W}(z)=\frac{\bar{q}_{L}^{2}}{\bar{k}_{L}} \bar{A} e^{-\bar{k}_{L}|z|}=-\frac{b \bar{q}_{L}^{2}}{2(\lambda+2 \mu)} \frac{J_{+}\left(k_{L}, d\right) e^{-\bar{k}_{L}(|z|-d)}}{F(\omega, q)},
\end{aligned}
$$

where the factor $F(\omega, q)=\bar{k}_{L} \cosh k_{L} d+k_{L} \sinh k_{L} d$ is introduced. In the absence of interaction $(b=0$ or $n=0)$, the set of $\mathrm{BC}$ equations is uniform, and the equality of the determinant to zero (A 2.2) determines the law of dispersion of free waves able to propagate in our composite medium. The condition $k_{T}=0$ determines the transverse wave which in our model $(\bar{\mu}=\mu)$ remains to be 


\section{A. A. Demidenko et al.: Amplification of localized acoustic waves by ...}

bulk-type. The condition $F(\omega, q)=0$ determines the dispersion law of the longitudinal localized wave which decays outside the layer as $e^{-\bar{k}_{L}|z|}$. Therefore, the condition $\bar{k}_{L}^{2}=q^{2}-\bar{q}_{L}^{2}>0$ should hold, and $q>\bar{q}_{L}$. The factor $F$ can turn to zero only if $k_{L}^{2}=q^{2}-q_{L}^{2}<0$, i.e. $q<q_{L}$. Hence, $\bar{q}_{L}<q_{L}=\sqrt{h} \bar{q}_{L}$. Thus, the localized wave is possible only when the medium inside the layer is «softer» than that outside, i.e.

$h=\frac{\bar{\lambda}+2 \mu}{\lambda+2 \mu}>1$.

In the limiting case of vacuum inside the layer, the wave is converted into two independent Rayleigh waves in the upper and lower half-spaces.

The dispersion equation for the localized free wave can be rewritten as:

$$
F(\omega, q)=\sqrt{q^{2}-\bar{q}_{L}^{2}} \cos d \sqrt{q_{L}^{2}-q^{2}}-\sqrt{q_{L}^{2}-q^{2}} \sin d \sqrt{q_{L}^{2}-q^{2}}=0 .
$$

This equation has an infinite number of solutions (branches) $\omega(q)$. All branches are located on the plane $\omega(q)$ between the straight lines $\omega=V_{L} q$, i.e. the dispersion law of the longitudinal wave in the inner medium, and the line $\omega=\bar{V}_{L} q$ corresponding to the bulk wave in the outer medium.

The lowest (zero) branch (at $(h-1) q d<<1$ ) has the following form:

$\omega \approx \bar{V}_{L} q\left[1-\frac{1}{2} q^{2} d^{2}(h-1)^{2}\right]$.

The origin points of the subsequent branches are determined by the values of $q$ and $q_{L}$ :

$q_{v 0}=\frac{1}{\sqrt{h-1}} \frac{v \pi}{d} ; \quad q_{L, v 0}=\sqrt{\frac{h}{h-1}} \frac{v \pi}{d} ; v=1,2 \ldots$

The behavior of the $V$-th branch near the point of its origin can be also easily found:

$$
\omega_{v}=\bar{V}_{L}\left[q_{v 0}+\left(q-q_{v 0}\right)-\frac{1}{2} q_{v 0} d^{2}(h-1)^{2}\left(q-q_{v 0}\right)^{2}+\ldots\right]
$$

$q>q_{v 0}$.

At large $q$, all branches asymptotically approach the straight line $q=q_{L}$. Figure 1 shows, for illustration, the zero branch and five subsequent branches for the case of a strong difference in the elastic properties $(h=2)$.

Let us estimate quantitatively the frequencies corresponding to the points of origin of non-zero branches for the case of our interest, with thin layers $(2 d \sim 100 \AA)$ and the difference in elastic properties of $10 \%$, i.e. $h=1.1$ :

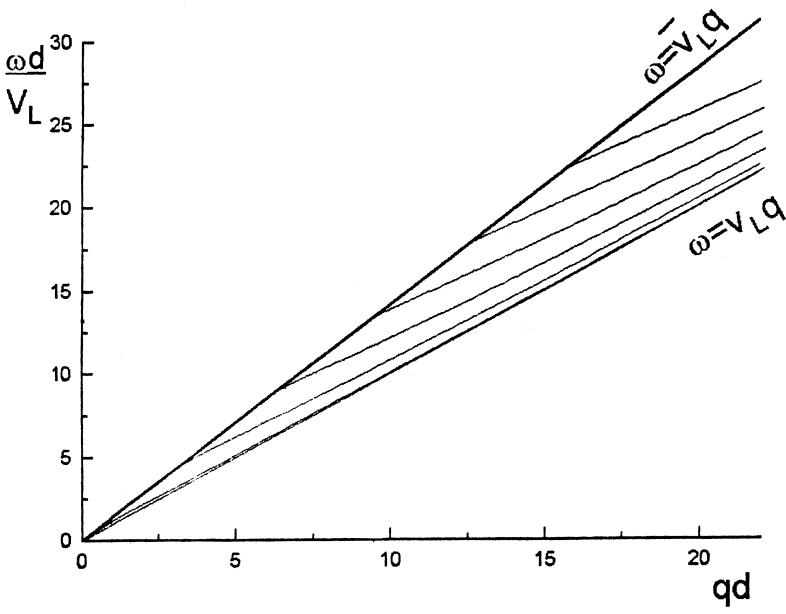

Fig. 1. The zero branch and five subsequent branches of the dispersion law of the localized acoustic waves at $h=2$ according to Eq.(12).

$\omega_{10}=\bar{V}_{L} q_{10}=\frac{\bar{V}_{L} \pi}{\sqrt{h-1}} \frac{1}{d} \sim 10^{13} s^{-1}$,

which corresponds to the THz- range. Analysis of the electron-phonon interactions at such high frequencies requires a quantum approach, so for the case of thin layers we shall deal only with the zero branch $\omega(q)$. Nonzero modes in the general case are considered in [19, 20].

For the zero branch and small thicknesses $\left(d \sqrt{q_{L}^{2}-q^{2}}<<1\right)$ instead of (12) we have:

$F \equiv \bar{k}_{L}-q^{2}(h-1) d=0$

At a fixed frequency, the solution of this equation is:

$q_{0}=\bar{q}_{L}\left[1+\frac{1}{2} \bar{q}_{L}^{2}(h-1)^{2} d^{2}\right]$

which is inversion of Eq.(13). It is worth noting that the wave localization near the layer $\bar{k}_{L}$, increases quadratically with the frequency. Obviously, it follows from (16) that

$\bar{k}_{L}=q^{2}(\omega)(h-1) d \approx \frac{\omega^{2}}{V_{L}^{2}}(h-1) d$.

In the case of a non-zero interaction with the carriers, the right-hand side $R(q)$ appears in Eq.(16), which in the general case is complex. We assume it to be small in comparison to each terms in the left-hand side. The form of $R(q)$ will be specified below. As a result, at a 


\section{A. A. Demidenko et al.: Amplification of localized acoustic waves by ...}

fixed frequency $\omega$, the index of spatial decay $\bar{k}_{L}$ and the wave vector $q$ will be modified:

$\bar{k}_{L}=q_{0}^{2}(h-1) d+R\left(q_{0}\right)$;

$q-q_{0}=R\left(q_{0}\right)\left[q_{0}(h-1) d+\frac{R\left(q_{0}\right)}{2 q_{0}}\right]$.

The determinant of the set of $\mathrm{BC}$ equations is now different from zero, and the above solutions of the nonuniform equations are valid.

In Eq.(19), the first term describes the localization caused by nonuniformities of the medium, and the second one corresponds to the localization due to interaction with electrons reported previously [22, 23]. If the medium is uniform $(h=1)$ and there is no interaction $(R=0)$, then $\bar{k}_{L}=0$, the dependence of $\bar{W}(10)$ on $z$ disappears, i.e,. we have the case of a uniform plane bulk wave propagating along the $X$ axis.

\section{Electron states}

In the previous section, it was assumed that the electron force acting on the lattice and the electron concentration are given. In this section, we will express these functions in the explicit form.

The electron states in the quantum well are described by the $3 \mathrm{D}$ Schrödinger equation:

$$
\hat{H}_{0} \Psi_{i, \vec{\kappa}}(\vec{r}, z)=E_{i, \vec{k}} \Psi_{i, \vec{\kappa}}(\vec{r}, z),
$$

where the Hamiltonian $\hat{H}_{0}$ includes the kinetic energy of electrons and the potential of the heterostructure $V(z)$ creating the quantum well. $\Psi_{i, \bar{\kappa}}(\vec{r}, z)$ and $E_{i}, \bar{k}$ denote the wave function and the electron energy in the $i$-th twodimensional subband with a planar wave vector $\vec{k}, \vec{r}$ is the in-plane radius-vector:

$$
\Psi_{i, \vec{k}}(\vec{r}, z)=\frac{1}{\sqrt{S}} e^{i \vec{k} \vec{r}} \chi_{i}(z) ; \mathrm{E}_{i, \bar{k}}=\epsilon_{i}+\frac{\hbar^{2} k^{2}}{2 m} .
$$

Here, $S$ is the area of the layer with the quantum well, the wave function of transverse motion $\chi_{i}(z)$ is normalized by unity, $\in_{i}$ is the energy of the bottom of the $i$ th subband, and $m$ is the effective mass.

In the presence of an acoustic wave, we should include in the total Hamiltonian of the system the potential energy consisting of the electrostatic energy and that of the electron-phonon interaction:

$$
\Phi(\vec{r}, z, t)=e \phi(\vec{r}, z, t)+b W(\vec{r}, z, t) .
$$

The dependence of $\Phi$ on $\vec{r}$ and $t$ is assumed to be proportional to $e^{i k \vec{r}-i \omega t}$. The self-consistent electrical potential $\phi$ is determined by the Poisson equation $\phi^{\prime \prime}-q^{2} \phi=-\frac{4 \pi e}{\varepsilon} n(z)$

where $e$ is the charge of the carriers. Taking into account that outside the layer $\bar{n}(z)=0$, and using the BCs at the layer boundary-continuity of $\phi$ and $\frac{\partial \phi}{\partial z}(\bar{\varepsilon}=\varepsilon)$ we obtain:

$$
\begin{aligned}
& \phi=\frac{4 \pi e}{2 \varepsilon q}\left[e^{-q d}\left(J_{+}(q, d)+J_{-}(q, d)\right) \cosh q z-J_{-}(q, z)\right] ;|z|<d, \\
& \bar{\phi}=\frac{4 \pi e}{2 \varepsilon q}\left[J_{+}(q, d) \cosh q d-J_{-}(q, d) \sinh q d\right] e^{-q|z|} ;|z\rangle d .
\end{aligned}
$$

When considering the kinetics of electrons in the field $\Phi(\vec{r}, z, t)$, we restrict ourselves to the case when longitudinal motion of electrons can be approximated quasi-classically. The relevant kinetic equations can be simplified for the next limiting cases: (1) when the single lowest subband $i=1$ is occupied (the quantum limit, $\mathrm{QW}$ ); (2) when the number of occupied subbands is so large that transverse motion can be also described quasi-classically - the case of the classical well $(\mathrm{CW})$. It is shown in Appendix III how to introduce the electron distribution function in these cases. The charge carriers are subjected to the action of the resulting self-consistent (threedimensional) effective field

$\bar{E}_{3 D}^{*}=-\nabla \phi-\frac{b}{e} \nabla W$

\section{Quantum limit $(Q W)$}

If the lowest subband is occupied and the distance to the next subbands are so large that the potential (23) does not cause any inter-subband transitions, then the quasiclassical distribution of electrons can be characterized by a single function $F_{(2 D)}(\vec{r}, \vec{V}, t), \vec{V}=\frac{1}{m} \hbar \vec{k}$, satisfying the kinetic equation. This, according to Appendix III, can be written in the standard form [32]:

$$
\frac{\partial F_{(2 D)}}{\partial t}+\vec{V} \cdot \frac{\partial F_{(2 D)}}{\partial \vec{r}}-\frac{1}{m} \frac{\partial \Phi_{(2 D)}}{\partial \vec{r}} \cdot \frac{\partial F_{(2 D)}}{\partial \vec{V}}=\left[\frac{\partial F_{(2 D)}}{\partial t}\right]_{\text {coll }} .
$$

The potential energy of the $2 \mathrm{D}$ carriers is introduced here as:

$\Phi_{(2 D)}(\vec{r}, t)=\int d z\left|\chi_{1}(z)\right|^{2} \Phi(\vec{r}, z, t)$.

The distribution function $F_{(2 D)}$ determines the total $2 \mathrm{D}$ concentration of charge carriers: 


\section{A. A. Demidenko et al.: Amplification of localized acoustic waves by ...}

$$
\begin{aligned}
& N(\vec{r}, t)=N_{s}+n_{s}(\vec{r}, t)= \\
& =\frac{2}{S} \sum_{\vec{k}} F_{(2 D)}(\vec{r}, \vec{V}, t)=2\left(\frac{m}{2 \pi \hbar}\right)^{2} \int d^{2} V F_{(2 D)},
\end{aligned}
$$

where $n_{s}(\vec{r}, t)=n_{s} e^{i \vec{q} \vec{r}-i \omega t}$. Using $F_{(2 D)}$, one can determine the energy of the electron-phonon interaction, i.e. the change in the energy of electrons in the deformation field, $\delta F_{\text {int }}$. Obviously, in the adiabatic approximation $\left(V>>V_{L, T}\right)$, the change of energy of a single quasi-classical electron at the point $\vec{r}$ due to deformation can be written similarly to Eq.(27):

$$
\delta E_{1, \vec{k}}(\vec{r}, t)=b \int d z\left|\chi_{1}(z)\right|^{2} \operatorname{div} \vec{U}(\vec{r}, z, t) .
$$

The change in the energy of all electrons is equal to

$$
\begin{aligned}
& \delta E_{\mathrm{int}}=\int d^{2} r \sum_{\vec{k}} \delta E_{1, \vec{k}}(\vec{r}, t) \frac{2}{S} F_{(2 D)}(\vec{r}, \vec{V}, t)= \\
& =b \int d^{2} r d z N(\vec{r}, t)\left|\chi_{1}(z)\right|^{2} \operatorname{div} \vec{U}(\vec{r}, z, t) .
\end{aligned}
$$

Therefore, the density of the energy of electronphonon interactions is equal to

$$
\delta \varepsilon_{\mathrm{int}}=b N(\vec{r}, t)\left|\chi_{1}(z)\right|^{2} \operatorname{div} \vec{U}(\vec{r}, z, t) .
$$

The density of the free energy of the entire system consists of the elastic deformation energy $\varepsilon_{E D}$, the electron energy $\varepsilon_{e}$, and of the electron-phonon interaction $\delta \varepsilon_{\text {int }}$ :

$$
\varepsilon=\varepsilon_{E D}+\varepsilon_{e}+\delta \varepsilon_{\mathrm{int}} .
$$

Using this relation, one can calculate the stress tensor:

$$
\frac{\partial \varepsilon}{\partial u_{i j}}=\frac{\partial \varepsilon_{E D}}{\partial u_{i j}}+\frac{\partial \delta \varepsilon_{\text {int }}}{\partial u_{i j}}=\sigma_{i j}^{E D}+\delta_{i j} b\left|\chi_{1}(z)\right|^{2} N(\vec{r}, t) .
$$

The equilibrium two-dimensional concentration $N_{s}$ included in $N(\vec{r}, t)$ results only in a static deformation, which will not be considered here. In section II, we introduced a non-equilibrium addition to the $2 \mathrm{D}$ concentration in the form $n(z) e^{i \vec{q} \vec{r}-i \omega t}$. According to (28) and (31), the amplitude of this dependence, present in the equation of lattice motion, is

$$
n(z)=n_{s}\left|\chi_{1}(z)\right|^{2}
$$

Thus, we obtain a self-consisted set of equations: equation for sound (1), (2) and the Poisson equation with the right-hand sides determined by (32), the kinetic equation (26) with the potential energy $\Phi_{(2 D)}$, determined from equations (23), (27), and the relation (28) for the $2 \mathrm{D}$ carrier concentration.

The kinetic equation (26) for the 2D distribution function $F \equiv F_{2 D}$ will be solved in the approximation of the relaxation time. Taking into account the assumed form of dependence on $\vec{r}$ and $t$, we will rewrite (26) as follows:

$$
\begin{aligned}
& i(-\omega+\vec{q} \cdot \vec{V}) F+\frac{e}{m}\left(\vec{E}^{*}+\vec{E}_{0}\right) \cdot \frac{\partial F}{\partial \vec{V}}= \\
& =-\frac{1}{\tau}\left[F-F_{0}\left(\vec{V}-\dot{\vec{U}} \mid N_{s}+n_{s} e^{i \vec{q} \vec{r}-i \omega t}\right)\right],
\end{aligned}
$$

where $\vec{E}^{*}=-\frac{1}{e} \frac{\partial}{\partial \vec{r}} \Phi_{2 D}$ is the effective field of the sound wave acting on the $2 \mathrm{D}$ electrons (see Appendix III), $\vec{E}_{0}$ is the constant drift field, and $\tau$ is the relaxation time. The argument of the equilibrium function $F_{0}$ corresponds to the shift of the center of the velocity distribution by the velocity of the scattering impurities $\vec{U}$ ( $\vec{U}$ is the elastic displacement vector of the medium). However, below we will neglect this shift, because it is essential only at low frequencies $\omega<10^{6} s^{-1}$. Moreover, as shown in [28], this contribution gives rise to a term in the electron current which exactly compensates the ion current. At high frequencies, we can neglect both, this shift and the ion current, and assume the total current to be equal to the electron one. $F_{0}$ also depends on the total 2D concentration $N(\vec{r}, t)$ parametrically. Thus, now the equilibrium function $F_{0}$ is the Fermi function of the total energy $\varepsilon+\epsilon_{1}=\frac{m V^{2}}{2}+\epsilon_{1}$, where $\epsilon_{1}$ is the energy of the bottom of the lowest subband occupied. The chemical potential is calculated through the total $2 \mathrm{D}$ concentration of electrons.

In the equilibrium state, according to (28), for degenerate electrons (the degeneration temperature at the parameter values chosen below is $\sim 100 \mathrm{~K}$ ) we have

$$
\begin{aligned}
& N_{s}=2\left(\frac{m}{2 \pi \hbar}\right)^{2} \int d^{2} V F_{0}=\frac{m}{\pi \hbar^{2}} \int_{0}^{\infty} \frac{d \varepsilon}{\exp \left(\frac{\varepsilon+\epsilon_{1}-\varepsilon_{F}}{k_{B} T}\right)+1}= \\
& =\frac{m}{\pi \hbar^{2}}\left(\varepsilon_{F}-\epsilon_{1}\right) \equiv \frac{m}{\pi \hbar^{2}} \varepsilon_{F}^{\prime},
\end{aligned}
$$

where $\varepsilon_{F}$ is the chemical potential. Therefore, the Fermi energy is equal to the difference $\varepsilon_{F}-\epsilon_{1}=\varepsilon_{F}^{\prime}$ related to the equilibrium $2 \mathrm{D}$ concentration as

$\varepsilon_{F}^{\prime}=\frac{\pi \hbar^{2}}{m} N_{s}$.

Then, the Fermi velocity $V_{F}$ and the Fermi electron wavelength $\lambda_{F}$ are 


\section{A. A. Demidenko et al.: Amplification of localized acoustic waves by ...}

$V_{F}=\frac{\hbar}{m} \sqrt{2 \pi N_{s}}, \quad \lambda_{F}=\sqrt{\frac{2 \pi}{N_{s}}}$.

The kinetic equation (33) will be solved in the linear approximation with respect to small deviations $E^{*}$ and $n_{s}$, and linearly with respect to the drift field $E_{0}$. Thus we assume the latter to be weak (the heating of the electron gas is neglected, and the electron drift velocity $V_{d}=\mu E_{0}=\frac{e \tau}{m} E_{0}$ is constant). The right-hand side of (33) can be expanded as

$$
\begin{aligned}
& F_{0}\left(\varepsilon \mid N_{s}+n_{s}\right)=F_{0}\left(\varepsilon \mid N_{s}\right)+\frac{\partial F_{0}}{\partial N_{s}} n_{s}= \\
& =F_{0}\left(\varepsilon \mid N_{s}\right)+\frac{\partial F_{0}}{\partial \varepsilon_{F}^{\prime}} \frac{\partial \varepsilon_{F}^{\prime}}{\partial N_{s}} n_{s}=F_{0}\left(\varepsilon \mid N_{s}\right)-\frac{\pi \hbar^{2}}{m} n_{s} \frac{\partial F_{0}}{\partial \varepsilon} .
\end{aligned}
$$

The non-equilibrium distribution function can be written as

$F=F_{0}+F_{1}^{0}+F_{1}^{1}$,

where $F_{1}^{0}(\vec{V})$ is the non-equilibrium additive associated with the drift in the external field $E_{0}$, independent of $\vec{r}$ and $t, F_{1}^{1}(\vec{V}, \vec{r}, t)$ is a small non-equilibrium additive due to the effective field $E^{*}$ and the non-equilibrium concentration $n_{s}$. It is easy to obtain for $F_{1}^{0}$ that

$F_{1}^{0}=-m \frac{\partial F_{0}}{\partial \varepsilon} \vec{V}_{d} \cdot \vec{V}$

Using (36) and (37) and applying the standard linearization procedure for Eq.(33), we obtain for $F_{1}^{1}$ :

$$
\begin{aligned}
& F_{1}^{1}=\left(-\frac{\partial F_{0}}{\partial \varepsilon}\right)\left\{e \tau\left[\frac{\vec{V}}{Q}+\vec{V}_{d}\left(\frac{1}{Q}+\frac{1}{Q^{2}}\right)+\frac{i \tau\left(\vec{q} \cdot \vec{V}_{d}\right) \vec{V}}{Q^{3}}\right] \cdot \vec{E}^{*}+\right. \\
& \left.+\frac{\pi \hbar^{2}}{m}\left[\frac{1}{Q}+\frac{i \tau\left(\vec{q} \cdot \vec{V}_{d}\right)}{Q^{3}}\right] n_{s}\right\}+ \\
& +\frac{\partial^{2} F_{0}}{\partial \varepsilon^{2}}\left[e \tau m\left(\vec{V} \cdot \vec{V}_{d}\right)\left(\frac{1}{Q}+\frac{1}{Q^{2}}\right)\left(\vec{V} \cdot \vec{E}^{*}\right)+\pi \hbar^{2} \frac{\left(\vec{V}_{d} \cdot \vec{V}\right)}{Q^{2}} n_{s}\right]
\end{aligned}
$$

where $Q$ denotes the following expression:

$$
Q=1-i \tau(\omega-\vec{q} \vec{V})
$$

The linear density of the $2 \mathrm{D}$ current is:

$$
\vec{j}=2 e\left(\frac{m}{2 \pi \hbar}\right)^{2} \int \vec{V} F(\vec{V}) d^{2} V .
$$

Taking into account the possibility of substituting in the integrand

$\vec{V} \frac{\partial^{2} F_{0}}{\partial \varepsilon^{2}} \rightarrow\left(-\frac{\partial F_{0}}{\partial \varepsilon}\right) \frac{1}{m} \frac{\partial}{\partial \vec{V}}$,

and the delta-like behavior of the derivative $\left(-\frac{\partial F_{0}}{\partial \varepsilon}\right)$, the current density $I_{x}$ in the longitudinal case, when the vectors $E^{*}, \vec{E}_{0}$ and $\vec{q}$ are directed along the $X$ axis, can be written as:

$$
\begin{aligned}
& j_{x}=\frac{\sigma_{s}}{\pi} E^{*}\left\{I_{21}+\frac{V_{d}}{V_{F}}\left[I_{11}+I_{12}-i p\left(I_{22}+I_{23}\right)\right]\right\}+ \\
& \frac{e n_{s} V_{F}}{2 \pi}\left\{I_{11}+\frac{V_{d}}{V_{F}}\left[I_{02}-i p I_{13}\right]\right\}
\end{aligned}
$$

where the angle integrals are denoted as:

$$
I_{n m}=\int_{0}^{2 \pi} \frac{\cos ^{n} \phi d \phi}{(a+i p \cos \phi)^{m}} .
$$

Here the following notations are introduced:

$\sigma_{s}=\frac{e^{2} \tau N_{s}}{m} ; p=q l=q \tau V_{F} ; a=1-i \omega \tau ; s=\sqrt{p^{2}+a^{2}}$.

At arbitrary $p$ and $\omega t$, the integrals in (41) can be easily obtained from the integral $I_{01}=\frac{2 \pi}{s}$. They are equal to:

$I_{11}=\frac{2 \pi}{i p}\left(1-\frac{a}{s}\right) ; \quad I_{21}=\frac{2 \pi a}{p^{2}}\left(1-\frac{a}{s}\right) ; \quad I_{12}=\frac{2 \pi p}{i s^{3}} ;$

$I_{22}=-\frac{2 \pi}{p^{2}}+\frac{2 \pi a}{p^{2} s^{3}}\left(2 p^{2}+a^{2}\right) ; I_{02}=\frac{2 \pi a}{s^{3}} ; I_{13}=\frac{3 \pi p a}{i s^{5}} ;$

$I_{23}=\frac{2 \pi}{s^{5}}\left(-p^{2}+\frac{a^{2}}{2}\right)$.

The continuity equation of the in-plane current has the following form:

$j_{x}=e n_{s} V_{L}$.

Using (44), the current (41) can be presented as:

$j_{x}=\sigma^{*} E^{*}$.

The general form of the effective 2D conductance $\sigma^{*}$ at arbitrary $q l, \omega t$ can be easily obtained from the above equations. Let us write down $\sigma^{*}$ for the two limiting cases: 1) $q l<<1$, then it should be all the more so for 


\section{A. A. Demidenko et al.: Amplification of localized acoustic waves by ...}

$$
\begin{aligned}
& \omega \tau=\frac{V_{L}}{V_{F}} q l<<1: \\
& \sigma_{<}^{*}=\frac{\varepsilon}{4 \pi} \frac{2 V_{L}}{L q} \frac{1}{\left(2 \frac{V_{L}}{V_{F}} \frac{1}{q l} \gamma+i\right)} .
\end{aligned}
$$

2) $q l>1$ :

$$
\sigma_{>}^{*}=\frac{\varepsilon}{4 \pi} \frac{2 V_{L}}{L_{q}}\left(\frac{V_{L}}{V_{F}} \gamma-i\right)
$$

Here the drift parameter is introduced

$$
\gamma=1-\frac{V_{d}}{V_{L}}
$$

and the two-dimensional screening length $L: L=\frac{2 e D}{4 \pi \sigma_{s}}$, $D$ is the diffusion coefficient, which in the $2 \mathrm{D}$ case, according to the Einstein relation, is equal to: $D=\frac{\tau V_{F}^{2}}{2}$, hence

$L=\frac{\hbar^{2} \varepsilon}{2 e^{2} m}$.

Thus, $L$ depends only on the effective mass $m$. For GaAs $m=0,067 m_{0}$, where $m_{0}$ is the free electron mass and $L=0.5 \cdot 10^{-6} \mathrm{~cm}$. The effective electric field $E^{*}(45)$ is determined by the integral of Eq.(25) over the layer thickness taking into account (10) and (32), i.e. $E^{*}=-\frac{i q}{e} \Phi_{(2 D)}$ where $\Phi_{(2 D)}$ is determined from equations (27), (23).

The wave function of the transverse motion for the lowest subband is assumed to be:

$$
\chi_{1}(z)=\frac{1}{\sqrt{d}} \cos \frac{\pi z}{2 d} .
$$

Calculating the integral in (27) with the wave function (50) and the expressions for $W \equiv \operatorname{div} \vec{U}$ and the potential $\phi$ obtained earlier, taking into account the formulae $(\mathrm{A}, 1.5)$ for the effective field in the case of a thin layer we obtain:

$$
E^{*}=-i \frac{4 \pi e}{2 \varepsilon}\left[1-G \omega^{2} \frac{q}{F(q)}\right] n_{s},
$$

where a constant coefficient $G$ is introduced:
$G=\frac{\varepsilon b^{2}}{4 \pi e^{2} \rho V_{L}^{4}}$.

Substituting (44), (47), (51) into (45) and dividing by $n$, we obtain the dispersion equation at $q l>1$ :

$(L q+1+i \eta) F(q)=q G \omega^{2}(1+i \eta)$

with the following notation used for simplicity:

$\eta=\frac{V_{L}}{V_{F}} \gamma$

In the absence of the interaction, the right-hand side of (53) is equal to zero, and the equation determines two noninteracting waves: the 2D drift wave discussed previously in [29] and the localized acoustic wave which was considered above. In the presence of interaction, both of these waves do not represent the exact solutions, and mixed acoustic and charge waves emerge. If the interaction is weak, it can be treated in terms of the perturbation theory, as it was made above in section II for the acoustic wave. The righthand side of $R(q)$, introduced in (19) and (20), according to (53), is equal to:

$$
R(q)=q \frac{G \omega^{2}(1+i \eta)}{L q+1+i \eta}=q G \omega^{2} L q \frac{1+\frac{1+\eta^{2}}{L q}+i \eta}{(1+L q)^{2}+\eta^{2}} .
$$

For further consideration, it is reasonable to estimate the relative role of the two terms in (19), (20). Let the difference of the elastic moduli be 10 per cent, i.e. $h-1=0.1$, and the half-thickness of the layer is $d=$ $=100 \AA$. Then, as estimations show, the first term dominates up to frequencies $\omega \approx 10^{13} \mathrm{~s}^{-1}$. Keeping only the first term in (20) and using $q_{0} \approx \bar{q}_{L}$ from (17), we express the left-hand side of (20) as

$q-\bar{q}_{L}=\frac{\omega}{\bar{V}_{L}^{\prime}}+i \frac{\alpha}{2}-\frac{\omega}{\bar{V}_{L}}=-\frac{\bar{V}_{L}^{\prime}-\bar{V}_{L}}{\bar{V}_{L}} \frac{\omega}{\bar{V}_{L}}+i \frac{\alpha}{2}$

where $\bar{V}_{L}$ is the initial and $\bar{V}_{L}^{\prime}$ is the renormalized sound velocity outside the layer. According to (56), we have for the renormalization of velocity:

$\frac{\Delta \bar{V}_{L}}{\bar{V}_{L}} \equiv \frac{\bar{V}_{L}^{\prime}-\bar{V}_{L}}{\bar{V}_{L}}=-G \omega^{2}(q d)(h-1) \times$

$\times \frac{1+L q+\eta^{2}}{(1+L q)^{2}+\eta^{2}}, \quad q=\bar{q}_{L}$

The absorption (amplification) coefficient of the acoustic wave, $\alpha(\omega)$, can be found as 


\section{A. A. Demidenko et al.: Amplification of localized acoustic waves by ...}

$$
\begin{aligned}
& \alpha(\omega) \equiv 2 \operatorname{Im}\{q\}= \\
& =2 G q(q d)(L q) \omega^{2}(h-1) \frac{\eta}{(1+L q)^{2}+\eta^{2}}, \\
& q=\bar{q}_{L}(\omega)
\end{aligned}
$$

It is evident, that if the Cherenkov criterion is met $V_{d}>V_{L}$

$(h<0)$ we get the amplification of the acoustic waves localized near the quantum well: $\alpha<0$. Since $\bar{q}_{L} \infty \omega$, the absorption (amplification) coefficient increases monotonically with the frequency. When the drift velocity rises, $\alpha(\omega)$ also increases. At large supercritical values, $V_{d} \gg V_{L}$, it turns out that $\alpha \propto \frac{V_{d}}{V_{F}} \propto \frac{\mu E_{0}}{\sqrt{N_{0}}}$.

Formally, the equation (58) has the drift maximum at $\eta= \pm(1+L q)$ equal to:

$$
\alpha_{\max }= \pm G \omega^{2} q(q d)(h-1) \frac{L q}{1+L q}
$$

The lower sign corresponds to amplification. The maximum of (58) is reached at $\frac{V_{L}}{V_{F}}\left(1-\frac{V_{d}}{V_{L}}\right)=-(1+L q)$, which corresponds to drift velocities exceeding $V_{F}$. Obviously, under these conditions the anisotropic part of the distribution function (34) is not small, and our analysis is no longer applicable. Therefore, in estimations below, we will restrict ourselves to the drift fields $E_{\mathrm{o}}$ such that $V_{d}<V_{F}$.

For quantitative estimations, we will take the parameters of a single-crystal GaAs: $\rho=5.317 \mathrm{~g} / \mathrm{cm}^{3} ; V_{L}=$ $=4.713 \cdot 10^{5} \mathrm{~cm} / \mathrm{s} ; \varepsilon=12.85 ; m=0.067 m_{0}$. Assume also that $b=15 \mathrm{eV}, d=10^{-6} \mathrm{~cm} ; h=1.1$ Then, $G=$ $=10^{-26} \mathrm{~s}^{2}, L=0.5 \cdot 10^{-6} \mathrm{~cm}$. Let the frequency be $\omega=$ $=100 \mathrm{GHz}=6.28 \cdot 10^{11} \mathrm{~s}^{-1}$, then $q_{L} \approx 1.33 \cdot 10^{6} \mathrm{~cm}^{-1}, L q_{L} \approx$ $\approx 0.665$. Also, let the equilibrium concentration of electrons be $N_{s}=2 \cdot 10^{12} \mathrm{~cm}^{-2}$. Then, according to (35), $V_{F}=$ $=5.6 \cdot 10^{7} \mathrm{~cm} / \mathrm{s}, \lambda_{\mathrm{F}}=180 \amalg$. Beyond any doubt, these parameters correspond to the quantum limit for electrons; however, the wavelength of the sound wave $2 \pi / q=470 \amalg$ is greater than $\lambda_{F}$, i.e., the acoustic wave and its interaction with electrons can be considered using the classical approach. If we assume the mobility to be $\mu=$ $=10^{5} \mathrm{~cm}^{2} / \mathrm{V} \cdot \mathrm{s}$, then $\tau=4 \cdot 10^{-12} \mathrm{~s}$ and $l=\tau V_{F}=1.6 \cdot 10^{-4} \mathrm{~cm}$, i.e. it is a fortiori the case of $q l>>1$. The condition (59) is fulfilled in the fields higher than $4.7 \mathrm{~V} / \mathrm{cm}$. For the parameters listed above and the field of $100 \mathrm{~V} / \mathrm{cm}\left(V_{d}=\right.$ $=10^{7} \mathrm{~V} / \mathrm{cm}<V_{F}$ ) we find for the acoustic wave amplification $\alpha=-60 \mathrm{~cm}^{-1}$.

The renormalization of the sound velocity, according to (56), is negative and in our case is of the order of
$10^{-4}$. The spatial decay index of the localized acoustic wave in the transverse direction outside the layer is determined by Eq.(19). At the chosen numerical values the wave intensity decays by a factor of $e$ at distances of about 5 wavelengths.

Consider also the case of a uniform medium (in terms of the elastic properties). Then $h=1$ and the first terms in Eqs. (19) and (20) disappear. The real part of $R(q)$ determines the localization of the wave near the layer:

$$
\operatorname{Re}\left\{\bar{k}_{L}\right\}=\operatorname{Re}\{R(q)\}=G \omega^{2} q \frac{1+L q+\eta^{2}}{(1+L q)^{2}+\eta^{2}} .
$$

The localization increases rapidly with $\omega$. For the absorption (amplification) coefficient, according to (20), (55), we have:

$$
\alpha=2 \operatorname{Im}\left\{\frac{R^{2}}{2 q}\right\}=2\left(G \omega^{2}\right)^{2} q(L q) \frac{\eta\left(1+L q+\eta^{2}\right)}{\left[(1+L q)^{2}+\eta^{2}\right]} .
$$

For the parameters chosen above, we find $\alpha=3 \mathrm{~cm}^{-1}$. If we select a frequency of $160 \mathrm{GHz}$, the value of the amplification coefficient will be equal to that considered above for $h=1.1$.

The index of spatial decay in the transverse direction $\bar{k}_{L}$ (19) is now complex. The phase front of the wave is not flat: outside the layer the phase velocity is slightly declined to the layer at $V_{d}<V_{L}$ and in the opposite direction at $V_{d}>V_{L}$. The renormalization of the velocity $\frac{\Delta \bar{V}_{L}}{\bar{V}_{L}}$ is now by the order of magnitude lower than in the case of $h=1.1$.

\section{Classical potential well (CW)}

If a large number of subbands in the well are occupied, then transverse motion of electrons in the layer can be considered quasi-classically. The potential energy of the electron is the sum of the potential energy of the well and the potential energy of the electron in the field of the sound wave (23). The potential energy forming the well is assumed to be zero inside the layer and infinite outside of it. Also, we suggest that the dragging field $\vec{E}_{0} \| X$ is applied. The 3D distribution function of electrons $f(x, z, \vec{v}, t)$, where $\vec{v}$ is the $3 \mathrm{D}$ electron velocity, does not depend on $z$ inside the layer and is equal to zero outside the layer, which is possible in the case of the mirror reflection of electrons from the boundaries. The bulk concentration is determined by Eqs. (A 1.4). The kinetic equation for the function $f$ is written in the standard form in the relaxation time approximation. Let us integrate this equation with respect to $z$ from $-d$ to $d$. The integrals from odd functions of $z$ disappear; in particular, the term proportional to $z$-projection of the effective field (25) and 


\section{A. A. Demidenko et al.: Amplification of localized acoustic waves by ...}

$\frac{d f}{d z}$ become equal to zero. Introducing the distribution function

$$
F(x, \vec{v}, t) \equiv \int_{-d}^{d} f(x, z, \vec{v}, t) d z=2 d f(x, 0, \vec{v}, t)
$$

one can obtain for it the equation similar to (33) where, however, $\vec{v}$ is the $3 \mathrm{D}$ velocity. The solution of the kinetic equation for the function $F$ is completely the same as in the quantum case.

Finally, for the flat current in the $X$ direction at $\vec{E}_{0}\|\vec{q}\| X$ we obtain

$J_{x}^{\prime}=\frac{3}{2} \sigma_{s}\left\{I_{21}^{\prime}+\frac{V_{d}}{V_{F}}\left[I_{11}^{\prime}+I_{12}^{\prime}-i p\left(I_{22}^{\prime}+I_{23}^{\prime}\right)\right]\right\} E_{x}^{*}+$

$+\frac{e V_{F} n_{s}}{2}\left[I_{11}^{\prime}+\frac{V_{d}}{V_{F}}\left(I_{02}^{\prime}-i p I_{13}^{\prime}\right)\right]$

where the angle integrals are denoted as

$I_{n m}^{\prime}=\int_{-1}^{1} \frac{x^{n} d x}{(a+i p x)^{m}}$,

$\sigma_{s}=2 d \sigma=2 d \frac{e^{2} \tau}{m} N=\frac{e^{2} \tau}{m} N_{s}$, Fermi velocity $V_{F}$ is

three-dimensional: $V_{F}=\frac{h}{m}\left(\frac{3 N}{8 \pi}\right)^{1 / 3}$ unlike that in (35).

Other notations are the same. The effective field in the $X$ direction is

$$
E_{x}^{*}=-\frac{i q}{e}\left[\frac{1}{2 d} \int_{-d}^{d} \Phi(x, z, t) d z\right]
$$

where the potential energy $\Phi$ is determined by Eqs. (23) and (24). Since the classical well is, generally speaking, wider than the quantum one, we present here the result of integration in (64) for an arbitrary thickness $d$. Using (25), (A 2.3), (A 1.5), we obtain for the effective field:

$$
E_{x}^{*}=-i \frac{4 \pi e}{2 \varepsilon}\left[\left(\frac{1}{q d}-\frac{1-e^{-2 q d}}{2 q^{2} d^{2}}\right)-G \omega^{2} \frac{q}{F(q)}\left(\frac{\sin d \sqrt{q_{L}^{2}-q^{2}}}{d \sqrt{q_{L}^{2}-q^{2}}}\right)^{2}\right] n_{s}
$$

At $q d<<1$ this equation is transformed into (51). The general form of the effective conductance $\sigma^{*}$ at arbitrary $q l$ and $\omega \tau$ is obtained from (45), (44), (62). Let us present $\sigma^{*}$ for the cases:

$$
\sigma_{<}^{*}=\frac{\varepsilon}{4 \pi} \frac{2 V_{L}}{\mathrm{~L}^{*} \mathrm{q}} \cdot \frac{1}{\frac{3 V_{L}}{V_{F}} \frac{1}{q l} \gamma+i}, \quad q l<<1
$$

$$
\sigma_{>}^{*}=\frac{\varepsilon}{4 \pi} \frac{2 V_{L}}{\mathrm{~L}^{*} \mathrm{q}} \cdot\left(\frac{\pi}{2} \frac{V_{L}}{V_{F}} \gamma-i\right), \quad q l>1
$$

Here, by analogy with the QW case, a parameter with the dimensionality of length is introduced:

$\mathrm{L}^{*}=\frac{1}{3 d} L_{3 D}^{2}$

$L_{3 D}$ is the $3 \mathrm{D}$ Debye screening length:

$L_{3 D}^{2}=\frac{\hbar^{2} \varepsilon}{4 \pi e^{2} m}\left(\frac{3}{8 \pi}\right)^{2 / 3} N^{-1 / 3}$.

We should note that the expression (66) for $q l<<1$ corresponds to the hydrodynamic approximation, when the bulk current density is determined by the equation $\vec{J}=\sigma \vec{E}^{*}+e \vec{V}_{d} n-e D \nabla n$. The results (66), (67) are analogous to those obtained earlier in [30] for the bulk waves. Using the expression (51) for the effective field at small thicknesses, one can easily come to the expression for $\alpha(\omega)$ in the form (58), where the substitution $L \rightarrow L^{*}$ (68) should be made.

\section{Discussion of the results}

Above we have analyzed the acoustic waves localized near the layer with a quantum well for charge carriers. The problem was considered for a simple model of an elastic isotropic medium. It was assumed that only one elastic constant - the first Lame constant - in the layer may be different from that in the surrounding material. In such model, the localization appears if the layer is «softer» than the ambient. If the elastic properties of the medium are uniform, the localization arises due to the deformation electron-phonon interaction. In both cases, the localization increases with the frequency of the acoustic waves.

We have considered the electron-phonon interaction via the deformation potential. The electrons in the quantum well interact with all possible acoustic waves; however, they affect most of the localized waves. The electron drift along the layer results in a slight renormalization of the wave velocities and leads to their attenuation or amplification. The necessary condition for amplification of the localized waves is the same as the well-known condition for Cherenkov radiation (59). A high mobility of carriers in the quantum wells results in low values of the electric fields required to satisfy this condition. If the amplification condition is satisfied, the magnitude of amplification rises essentially with frequency $\left(\alpha \propto \omega^{5}\right)$. The significant factor here is the increase of degree of wave localization with frequency: $\bar{k}_{L} \propto \omega^{2}$. Along with the acoustic wave, the oscillations of the electrostatic potential and the charge density of the 2D electrons are amplified. Different relations between the frequency $\omega$ 


\section{A. A. Demidenko et al.: Amplification of localized acoustic waves by ...}

and electron relaxation time $\tau$, between the wave vector $q$ and the free length $l$ were analysed. It was found that in the most important case of $q l \gg 1$ at frequencies in the range of $100 \mathrm{GHz}$ the amplification coefficient of the localized acoustic wave can reach the values of tens of inverse $\mathrm{cm}$. The equations obtained indicate that amplification continues to rise with frequency. However, at higher frequencies the quantum treatment of longitudinal electron motion is needed.

From the very beginning, we did not take into account in the equations the lattice attenuation of sound, since in the lowest approximation in respect to dissipation, which is the only approximation considered here, all dissipation mechanisms are additive. In the perfect crystals of the GaAs type the losses of acoustic waves are much less than $10 \mathrm{~cm}^{-1}$ up to the frequencies above 1 $\mathrm{THz}$ [31]. Thus, drift-induced amplification obtained is much greater than the lattice sound attenuation, at least in perfect crystals. The amplification factor does not depend strongly on whether only the lowest subband is occupied (the quantum limit), or many subbands are occupied (the classical well).

It is of interest to compare the results obtained with the calculation of the amplification coefficient in the bulk material [30]:

$\alpha_{3 D}=\frac{m b^{2} \omega}{6 \pi \hbar^{3} \rho V_{L}^{2}} \times \frac{\left(q L_{3 D}\right)^{4}}{\left(1+\left(q L_{3 D}\right)^{2}\right)} \times\left(1-\frac{V_{d}}{V_{L}}\right)$

where all notations are the same as in the previous section. The amplification coefficient for the electron drift in the quantum well (58) for the practically important conditions $q L<1,|\eta|<1$ can be rewritten in a similar manner:

$$
\begin{aligned}
& \alpha=\frac{m b^{2} \omega}{2^{3 / 2} \pi^{5 / 2} \hbar^{3} \rho V_{L}^{2}}(h-1) \times \\
& \times \frac{q}{N_{s}^{1 / 2}}(q d)(q L)^{2} \times\left(1-\frac{V_{d}}{V_{L}}\right) .
\end{aligned}
$$

In these equations, we have separated three factors: the first one depends on the elastic properties, the effective mass of electrons and the electron-phonon interaction; the second one is associated mainly with the screening, and the third one relates to the electron drift. Comparing the equations, we can conclude that the first factor in our case is lower by a coefficient of $(h-1)$. The second factor in the frequency range $\omega \sim 100 \mathrm{GHz}$ is of the order of unity. The third factor is of the same form in both cases. However, the mobilities of charge carriers in quantum heterostructures can be much higher than in the bulk material, so that the same supercritical condition $1-V_{d} / V_{L}$ can be reached at much lower electric fields.

Thus, we have shown that in the case of a parallel transport of electrons in the quantum well amplifica- tion of the high-frequency acoustic waves, localized near the well, is observed. This effect can be used for sources of intense and strongly collimated high-frequency acoustic oscillations. Such short-wave coherent oscillations can find a broad range of applications: generation of microwave radiation, non-destructive testing of microelectronic devices, modulation of optical signals, deflectors for laser beam control, etc.

The authors are grateful to Dr. V. N. Piskovoy and Dr. V. I. Pipa for discussions of some problems considered in this paper, and to E. V. Mozdor for assistance in manuscript preparation. This work was supported by the State Fund of Fundamental Research of Ukraine, Grant No. 2.4/679 and, in part, by STCU grant No. 437.

\section{Appendix I}

Let us define integrals:

$I^{(v)}(q, z)=e^{q z} \int_{0}^{z} e^{-q z} n^{(v)}(z) d z$

$J_{ \pm}^{(v)}(q, z)=I^{(v)}(q, z) \pm I^{(v)}(-q, z)$

where

$n^{(v)}=\frac{d^{v} n}{d z^{v}}$.

Integration by parts yields:

$I^{(v)}(q, z)=n^{(v-1)}(z)-e^{q z} n^{(v-1)}(0)+q I^{(v-1)}(q, z)$.

Then

$J_{+}^{(v)}(q, z)=2 n^{(v-1)}(z)-$

$-2 n^{(v-1)}(0) \cosh q z+q J_{-}^{(v-1)}(q, z)$

$J_{-}^{(v)}(q, z)=-2 n^{(v-1)}(0) \sinh q z+$

$+q J_{+}^{(v-1)}(q, z)=-2 n^{(v-1)}(0) \sinh q z+$

$+q\left[2 n^{(v-2)}(z)-2 n^{(v-2)}(0) \cosh q z+q J_{-}^{(v-2)}(q, z)\right]$.

The derivatives of the characteristic polynomial (1.4a) are:

$P^{\prime}\left( \pm k_{L}\right)= \pm 2 k_{L}\left(q_{T}^{2}-q_{L}^{2}\right) ; \quad P^{\prime}\left( \pm k_{T}\right)=\mp 2 k_{T}\left(q_{T}^{2}-q_{L}^{2}\right)$.

The sought function $y(z)(1.4)$ is equal to:

$y(z)=-\frac{\beta}{2 q_{\mathrm{T}}^{2}\left(q_{\mathrm{T}}^{2}-q_{L}^{2}\right)} \Phi(z) ;$ 


\section{A. A. Demidenko et al.: Amplification of localized acoustic waves by ...}

$\Phi(z)=\frac{1}{\kappa_{L}}\left[J_{-}^{(3)}\left(k_{L}, z\right)-k_{\mathrm{T}}^{2} J_{-}^{(1)}\left(k_{L}, z\right)\right]_{-}$

$-\frac{1}{k_{\mathrm{T}}}\left[J_{-}^{(3)}\left(k_{\mathrm{T}}, z\right)-k_{\mathrm{T}}^{2} J_{-}^{(1)}\left(k_{\mathrm{T}}, z\right)\right]$

Using the recurrent equations written above, taking into account that $n^{\prime}(0)=0$, we obtain the expression (6) in the main text. The integrals $J_{ \pm}(q, z) \equiv J_{ \pm}^{(0)}(q, z)$ are determined as:

$J_{ \pm}(q, z)=e^{q z} \int_{0}^{z} e^{-q z} n(z) d z \pm e^{-q z} \int_{0}^{z} e^{q z} n(z) d z$

The properties of these integrals are:

$$
\frac{d}{d z} J_{-}(q, z)=q J_{+}(q, z) ; \frac{d}{d z} J_{+}(q, z)=q J_{-}(q, z)+2 n(z) ;
$$

$\int_{0}^{z} J_{+}(q, z) d z=\frac{1}{q} J_{-}(q, z)$.

Expansion at small $z<<\frac{1}{q}$ yields:

$J_{-}(q, z)=q n(0) z^{2}+q\left[n^{\prime \prime}(0)+q^{2} n(0)\right] \frac{z^{4}}{12}+\ldots$

$J_{+}(q, z)=2 n(0) z+\left[n^{\prime \prime}(0)+q^{2} n(0)\right] \frac{z^{3}}{3}+\ldots$

For a classical potential well $(\mathrm{CW})$ :

$n(z)=\left\{\begin{array}{ll}n(0)=\frac{n_{s}}{2 d}=\text { const } & |z|<d \\ 0 & |z|>d\end{array}\right.$.

Then

$J_{+}(q, z)=n_{s} \frac{\sinh q z}{q d} ; J_{-}(q, z)=n_{s} \frac{(\cosh q z-1)}{q d}$.

For the quantum well (QW):

$$
n(z)= \begin{cases}\frac{n_{s}}{d} \cos ^{2} \frac{\pi z}{2 d} & |z|<d \\ 0 & |z|>d\end{cases}
$$

In this case, if, for the narrow well, $(q d)^{2}$ is neglected in comparison to $\pi^{2}, J_{+}$and $J_{-}$are determined by the same equations (A 1.5).

\section{Appendix II}

The boundary conditions at $z=d$ (at $z=-d$ the conditions are identical because the functions are even):
1) $\bar{U}_{x}=U_{x}$;
2) $\bar{U}_{z}=U_{z}$;
3) $\bar{\sigma}_{x z}=\sigma_{x z}$;
4) $\bar{\sigma}_{z z}=\sigma_{z z} ; \mathrm{z}=\mathrm{d}$.

Taking into account that $\bar{\mu}=\mu$, and also that $\mathrm{n}( \pm \mathrm{d})$ $=0$, the 3 rd and 4 th conditions can be simplified:

3) $\frac{\partial \bar{U}_{x}}{\partial z}=\frac{\partial U_{x}}{\partial z}$;4) $h \bar{W}=W ; z=d$

The set of BC equations may be expressed in the following form:

$$
\left\{\begin{array}{l}
\bar{A} \frac{q^{2}}{\bar{k}_{L}} e^{-\bar{k}_{L} d}+\bar{B} k_{\mathrm{T}} e^{-k_{\mathrm{T}} d}+A \frac{q^{2}}{k_{L}} \cosh k_{L} d+B k_{T} \cosh k_{T} d= \\
\bar{A} e^{-\bar{k}_{L} d}+\bar{B} e^{-k_{T} d}-A \sinh k_{L} d-B \sinh k_{T} d=y(d) ; \\
\bar{A} e^{-\bar{k}_{L} d}-A \sinh k_{L} d=\frac{1}{q_{T}^{2}}\left[\psi^{\prime}(d)-k_{T}^{2} y(d)\right] \\
\bar{A} \frac{1}{\bar{k}_{L}} e^{-\bar{k}_{L} d}+A \frac{1}{k_{L}} \cosh k_{L} d=\frac{1}{q_{L}^{2}}\left[y^{\prime}(d)-\psi(d)-C\right]
\end{array}\right.
$$

The determinant of the system (A 2.1) is:

$$
\Delta=-\frac{k_{T}}{\bar{k}_{L} k_{L}} e^{-\bar{k}_{L} d} F
$$

$F=\bar{k}_{L} \cosh k_{L} d+k_{L} \sinh k_{L} d$.

Assuming $\Delta \neq 0$, all amplitudes can be determined from the system (A 2.1). For $W(z) \equiv \operatorname{div} \bar{U}$ it is sufficient to know only $A$ and $\bar{A}$, which are determined from the 3rd and 4th equations (A 2.1). As a result, we get:

$$
\begin{aligned}
& W(z)=-\frac{q_{L}^{2}}{k_{L}} A \cosh \left(k_{L} z\right)+y^{\prime}-\psi-C= \\
& =-\frac{\beta}{q_{T}^{2}}\left[\frac{q_{L}^{2}}{2} \frac{J_{+}\left(k_{L}, d\right)+\frac{\bar{k}_{L}}{k_{L}} J_{-}\left(k_{L}, d\right)}{F} \cosh k_{L} z+\right.
\end{aligned}
$$

$$
\left.+n(z)-\frac{q_{L}^{2}}{2 k_{L}} J_{-}\left(k_{L}, z\right)\right]
$$




\section{A. A. Demidenko et al.: Amplification of localized acoustic waves by ...}

$\bar{W}(z)=\frac{\bar{q}_{L}^{2}}{\bar{k}_{L}} \bar{A} e^{-\bar{k}_{L}|z|}=$

$=-\frac{\beta}{q_{T}^{2}} \frac{\bar{q}_{L}^{2}}{2} \frac{J_{+}\left(k_{L}, d\right) \cosh \left(k_{L} d\right)-J_{-}\left(k_{L}, d\right) \sinh \left(k_{L} d\right)}{F} \times$

$\times e^{-\bar{k}_{L}(|z|-d)}$.

Since we are interested in the range of wave vectors near the resonance, where $F$ is small, non-resonant terms can be neglected in (A 2.3) up to frequencies $\omega \approx 10^{13} \mathrm{~s}^{-1}$. Neglecting also the small terms $(q d<1)$ in the numerators of the fractions in (A 2.3), (A 2.4), we get the equations (10) from the main text.

\section{Appendix III}

The quasi-classical $N$-particle distribution function $f_{N}^{w}$ in a smooth field is obtained from the Wigner function

$$
\begin{aligned}
& f_{N}^{W}(q, p, t)= \\
& =\frac{1}{(2 \pi)^{3 N}} \int \frac{(2 \pi \hbar)^{3 N}}{V^{N}} d \vec{\gamma} \rho_{N}\left(\vec{q}+\frac{1}{2} \hbar \vec{\gamma}, \vec{q}-\frac{1}{2} \hbar \vec{\gamma}\right) e^{-i \vec{\gamma} \vec{p}}
\end{aligned}
$$

where $\hat{p}_{N}\left(q, q^{\prime}\right)$ is the density matrix in the $X$-presentation, $q \equiv \vec{r}_{1}, \vec{r}_{2} \ldots \vec{r}_{N}$, controlled by the equation

$$
i \hbar \frac{\partial \hat{p}_{N}}{\partial t}=\hat{H} \hat{\rho}_{N}-\hat{\rho}_{N} \hat{H} \text {, }
$$

$$
\hat{H} \equiv H\left(-i \hbar \frac{\partial}{\partial q}, q\right), N \text { is the number of electrons. The }
$$

limit of smooth (in respect to the de Broglie wavelength), fields is obtained at $\vec{\hbar} \rightarrow 0$ :

$$
\frac{\partial f_{N}}{\partial t}+\frac{\partial H}{\partial p} \frac{\partial f_{N}}{\partial q}+\frac{\partial H}{\partial q} \frac{\partial f_{N}}{\partial p}=\left[f_{N}\right]_{c o l l}
$$

where $H=H(p, q)$ is the classical Hamiltonian.

In the case of $2 \mathrm{D}$ electrons, it is convenient to use a mixed presentation for the density matrix: $p_{i, k, i^{\prime}, k^{\prime}}$, where $i, i^{\prime}$ are the numbers of subbands, $\vec{k}, \vec{k}^{\prime}$ are the 2D longitudinal wave vectors. In the coordinate presentation for the longitudinal variables

$$
\rho_{i, i^{\prime}}\left(\vec{r}, \vec{r}^{\prime}\right)=\sum_{k k} e^{-i \vec{k} \vec{r}} \rho_{i k, i k^{\prime}} e^{-i \vec{k}^{\prime} \vec{r}^{\prime}}
$$

The equation for this matrix has the form

$$
\begin{aligned}
& i \hbar \frac{\partial}{\partial t} \rho_{i, i^{\prime}}\left(\vec{r}, \vec{r}^{\prime}\right)= \\
& =\sum_{i^{\prime \prime}, \vec{r}^{\prime \prime}}\left[H_{i i^{\prime \prime}}\left(\vec{r}, \vec{r}^{\prime \prime}\right) \rho_{i^{\prime \prime}, i^{\prime}}\left(\vec{r}^{\prime \prime}, \vec{r}^{\prime}\right)-\rho_{i i^{\prime \prime}}\left(\vec{r}, \vec{r}^{\prime \prime}\right) H_{i^{\prime \prime}, i^{\prime}}\left(\vec{r}^{\prime \prime}, \vec{r}^{\prime}\right)\right]
\end{aligned}
$$

where $\sum_{\vec{r}^{\prime \prime}} \equiv \int d \vec{r}^{\prime \prime}$. If the only one subband $i=1$ is occupied and the transitions between the subbands are not essential, we have $H_{i, i^{\prime}} \sim \delta_{i, i^{\prime}}$. Then

$$
\begin{aligned}
& i \hbar \frac{\partial \rho_{11}\left(\vec{r}, \vec{r}^{\prime}\right)}{\partial t}= \\
& =\sum_{\vec{r}^{\prime \prime}}\left[H_{11}\left(\vec{r}, \vec{r}^{\prime \prime}\right) \rho_{11}\left(\vec{r}^{\prime \prime}, \vec{r}^{\prime}\right)-\rho_{11}\left(\vec{r}, \vec{r}^{\prime \prime}\right) H_{11}\left(\vec{r}^{\prime \prime}, \vec{r}^{\prime}\right)\right] .
\end{aligned}
$$

The latter equation is the complete analog of (A 3.1) with 2D $\vec{r}$ and $\vec{r}^{\prime}$ and the matrix elements of the 3D Hamiltonian $H_{0}+\Phi$. Here $H_{0}$ corresponds to (21), and the matrix elements are calculated for the functions $\chi_{1}(z)$ :

$$
\begin{aligned}
& H_{11}=<\chi_{1}\left|-\frac{\hbar^{2}}{2 m}\left(\Delta_{r}+\frac{d^{2}}{d z^{2}}\right)+V(z)+\Phi(\vec{r}, z)\right| \chi_{1}>= \\
& =\epsilon_{1}+\hat{H}^{(2 D)}(\vec{r}),
\end{aligned}
$$

$\hat{H}^{(2 D)}(\vec{r}) \equiv-\frac{\hbar^{2}}{2 m} \Delta_{r}+\left\langle\chi_{1} \Phi(\vec{r}, z) \chi_{1}\right\rangle$.

Using (A 3.3) and (A 3.4), in the limit $\hbar \rightarrow 0$ we obtain the standard equation (26) for the single-electron quasi-classical distribution function, where the velocity of $2 \mathrm{D}$ electrons is $\vec{V}=\partial H^{(2 D)} / \partial \vec{p}$, and the force acting on these electrons is equal to

$$
\begin{aligned}
& -\frac{\partial \hat{H}^{(2 D)}}{\partial \vec{r}}= \\
& =-\frac{\partial}{\partial \vec{r}}<\chi_{1}|\Phi(\vec{r}, z)| \chi_{1}>=-\frac{\partial \Phi_{(2 D)}}{\partial \vec{r}} .
\end{aligned}
$$

$\Phi_{(2 \mathrm{D})}$ is given by the integral (27).

\section{References}

1. K. B. Tolpygo, Z. I. Uritskii, K teorii podvizhnosti elektrona (On the theory of electron mobility) //Zhurn. Eksp. Teor. Fiz., 30, p.929 (1956) (in Russian).

2. D. L. White. Amplification of ultrasonic waves in piezoelectric semiconductors // J. Appl. Phys. 33, p.2547 (1962).

3. J. B. Gunn. Quantum theory of lattice-wave amplification in semiconductors // Phys. Rev. 138, p.1721 (1965).

4. Pustovoit V.I., Vzaimodeistvie elektronnykh potokov s uprugimi volnami reshetki (Interaction of electron flows with the elastic lattice waves) // Uspekhi Fiz. Nauk, 97, p.257 (1969) (in Russian).

5. V. L. Gurevich. Teoriya akusticheskikh svoistv piezokristallicheskikh poluprovodnikov (Theory of acoustic properties of piezo-crystal semiconductors) // Fiz. Tekhn. Polupr., 2, p.1557 (1968) (in Russian).

6. J. W. Tuckler, V. W. Rampton, Microwave Ultrasonics in Solid State Physics, North-Holland Publ. Co., Amsterdam, 1972.

7. S. I. Pekar, Elektron-fononnoe vzaimodeistvie proportsionalnoe vneshnemu prilozhennomu poliu i usilenie zvuka v poluprovodnikakh (Electron-phonon interaction proportional to the external field and sound amplification in semiconductors) // Zhurn. Eksp. 


\section{A. A. Demidenko et al.: Amplification of localized acoustic waves by ...}

Teor. Fiz., 49, p.621 (1965) (in Russian). A. A. Demidenko, S. I. Pekar, V. N. Piskovoi, B. E. Tsekvava, Ob usilenii ultrazvuka dreifovykh voln $\mathrm{v}$ poluprovodnikakh $\mathrm{v}$ elektricheskom i magnitnom poliakh (On amplification of ultrasound and drift waves in semiconductors in the electric and magnetic fields) // Zhurn. Eksp. Teor. Fiz., 52, p.710 (1967) (in Russian).

8. I. A. Viktorov, Zvukovye i poverkhnostnye volny v tverdykh telakh (Sound and surface waves in solids), Moscow, Nauka Publ., 1981(in Russian).

9. Yu. V. Guliaev, Poverkhnostnye ultrazvukovye volny v tverdykh telakh (Surface ultrasound waves in solids) // Pisma v Zhurn. Eksp. Teor. Fiz., 9, p.63 (1969) (in Russian).

10. C. Weisbuch, B. Vinter, Quantum Semiconductor Structures, Academic Press, San-Diego, 1991.

11. B. K. Ridley, Electrons and Phonons in Semiconductor Multilayers, Cambridge University Press, 1997.

12. B. K. Ridley, Electron-phonon Interaction in 2D Systems, in book «Hot Carriers in Semiconductor Nanostructures (Physics and applications)». Ed. J.Shaw, Academic Press, Boston, 1992, p. 17.

13. B. K. Redley, N. A. Zakhleniuk, Hot Electrons under Quantization Conditions. // J.Phys. Condens.Matter, 8, p.8525, (1996).

14. L. J. Challis, A. J. Kent, V. W. Rampton, Phonon Studies of TwoDimensional Electron Gas // Semicond.Sci. Technol. 5, 1179 (1990).

15. A. J. Kent, G. A. Hardy, P. Hawker, et al, Detection of Heat Pulses by the Two-Dimensional Electron Gas in a Silicon device / Phys. Rev. Lett., 61, p.180 (1988).

16. P. Hawker, A. J. Kent, 0. H. Hughes, L. J. Chillis, Changover from Acoustical to Optical Mode Phonon Emission by a Hot TwoDimensional Electron Gas in the GaAs/AIGaAs heterojunction / / Semicond.Sci. Technol., 7, 829 (1992)

17. M. Blencowe, A. Shik, Acoustoconductivity of Quantum Wires / / Phys. Rev., B. 54, p.13899 (1996).

18. L. Wendler, R. Houpt, V. G. Geigorjan, // Physica B 167, p.91 (1990) (in Russian).

19. L.Wendler, V.G. Grigorian. Acoustic interface waves in sandwich structures // Surf. Science 206, p.203 (1988) (in Russian).

20. V. G. Grigorian, L. Wender. Lokalizovannye akusticheskie volny $\mathrm{v}$ sloistykh strukturakh (Localized acoustic waves in layered structures) // Fiz. Tverdogo Tela 33, p.2120 (1991) (in Russian)

21. V. A. Kochelap, O. Gulseren. Confinement of acoustical phonons due to electron-phonon coupling in the electron sheet. // Sov. Phys. JETR, Letters, 56, n 2, p.94-98, (1992) (in Russian).

22. V. A. Kochelap, O. Gulseren. Localization of acoustical modes due to the electron-phonon interaction within two-dimensional electron gas // J.Phys. Condens. Matter 5, p.589 (1993) (in Russian).

23. V.A.Kochelap, V.V.Mitin. Effects of acoustic mode localization under dimentional crossover of an electron gas // Phys. Rev. B $\mathbf{5 5}$ p.10707 (1997) (in Russian)

24. C. Thomsen, H. T. Gruhn, H. J. Maris, J. Tauc, Picosecond Interferometric Technique for the Studies of Phonons in the Brillouin frequency range // Phys. Rev. B 34, p.4129 (1986) (in Russian).

25. H. T. Grahn, H. J. Maris, J. Taus, Picosecond Ultrasonics // IEEE Journal QE, 25, p.2561 (1989) (in Russian).

26. H. M. Lin, R. J. Stormer, H. J. Maris, Nondestructive Testing of Microstructures by Picosecond Ultrasonics // Journ. of Nondestructive Evaluation, 9, p.239 (1990) (in Russian).

27. L. D. Landau, E. M. Lifshits. Teoriya uprugosti (Theory of elasticity). Moscow, Nauka Publ., 1965.

28. B.E.Tsekvava. $\mathrm{K}$ teorii usileniya ultrazvuka $\mathrm{v}$ poluprovodnikakh (On theory of ultrasound amplification in semiconductors) // Fiz. Tekhn. Polupr., 6, p.1015 (1972) (in Russian).

29. B.K.Ridley. Space charge waves and the piezoelectric interaction in 2D semiconducting stryctures // Semicond. Sci. and Techn., 3, p.542 (1988) (in Russian).

30. H. Spector. Amplification of acoustic waves through interaction with conduction electrons // Phys. Rev. B 127, p.1084 (1962), Effect of an external electric field on the velocity of sound in semiconductors and semimetals, 134, p.A507 (1964) (in Russian).

31. S. Tamura, Spontaneous decay of LA phonons in quasi-isotopic solid // Phys. Rev. B, 31, 2574 (1985) (in Russian).

32. V. V. Mitin, V. A. Kochelap, M. A. Stroscio, Quantum Heterosrtuctures fot microelectronics and optoelectronics, Cambridge University Press, New York-Cambridge, 1998. 